Gut and Liver, Vol. 13 No. 4, July 2019, pp. 471-478

\title{
Safety Evaluation of Paclitaxel-Eluting Biliary Metal Stent with Sodium Caprate in Porcine Biliary Tract
}

\author{
Sung III Jang ${ }^{1}$, Seok Jeong ${ }^{2,3}$, Don Haeng Lee ${ }^{2,3}$, Kun Na ${ }^{4}$, Sugeun Yang ${ }^{5}$, and Dong Ki Lee ${ }^{1}$ \\ ${ }^{1}$ Department of Internal Medicine, Gangnam Severance Hospital, Yonsei University College of Medicine, Seoul, ${ }^{2}$ Department of Internal \\ Medicine, Inha University Hospital, Inha University College of Medicine, ${ }^{3}$ Utah-Inha DDS \& Advanced Therapeutics Research Center, Incheon, \\ ${ }^{4}$ Department of Biotechnology, The Catholic University of Korea, Bucheon, and ${ }^{5}$ World Class Smart Lab, Department of New Drug Development, \\ Inha University College of Medicine, Incheon, Korea
}

Background/Aims: Metallic stents designed to relieve malignant biliary obstruction are susceptible to occlusive tumor ingrowth or overgrowth. In a previous report, we described metallic stents covered with paclitaxel-incorporated membrane (MSCPM-I, II) to prevent occlusion from tumor ingrowth via antitumor effect. This new generation paclitaxeleluting biliary stent is further endowed with sodium caprate (MSCPM-III) for enhanced drug delivery. The purpose of this study is to examine the safety of its drug delivery system in the porcine biliary tract. Methods: MSCPM-III (10\% [wt/vol] paclitaxel) and covered metal stents (CMSs) were endoscopically inserted in porcine bile ducts in vivo. Histologic biliary changes, levels of paclitaxel released, and various serum analytes (albumin, alkaline phosphate, aspartate transaminase, alanine transaminase, total protein, total bilirubin, and direct bilirubin) were assessed. Results: Based on the intensity of reactive inflammation and fibrosis, changes in porcine biliary epithelium secondary to implanted MSCPMIII were deemed acceptable (i.e., safe). Histologic features in the MSCPM-III and CMS groups did not differ significantly. In a related serum analysis, paclitaxel release from MSCPMIII stents was below the limit of detection for 28 days. Biochemical analyses were also similar for the two groups, and no evidence of hepatic or renal toxicity was found in animals receiving MSCPM-III stents. Conclusions: In a prototypic porcine trial, this newly devised metal biliary stent incorporating both paclitaxel and sodium caprate appears to be safe in the porcine bile duct. (Gut Liver 2019;13:471-478)
Key Words: Drug-eluting stent; Self expandable metallic stent; Drug delivery systems; Biliary tract neoplasms; Pancreatic neoplasm

\section{INTRODUCTION}

Biliary decompression is an important palliative treatment for patients with unresectable malignant biliary obstruction. In this setting, endoscopic drainage typically is more useful than surgical bypass, ${ }^{1}$ and a self-expandable metal stent (SEMS) is more effective than a plastic stent. ${ }^{2}$ Although a covered SEMS has been developed to deter occlusive tumor ingrowth after placement, there has been no substantial improvement in terms of stent patency or migration stemming from either overgrowth/ ingrowth of tumor or biliary sludge.,

To prevent occlusive tumor ingrowth, we developed an antitumor drug-eluting biliary stent, ${ }^{5-11}$ which is a metallic stent covered paclitaxel (PTX)-incorporated membrane (MSCPM). Its safety was confirmed through animal ${ }^{5-7}$ and human studies, ${ }^{8-11}$ but its efficacy was unproven. This first-generation device failed to prevent tumor ingrowth, based on problematic biodegradation of polyurethane (PU) membranes, ${ }^{10}$ and the second-generation design (MSCPM-II) met with limited success in evaluating efficacy due to early stent occlusion (by food or sludge) or stent migration. ${ }^{11}$ However, there is a valid molecular rationale for combatting tumors through PTX-eluting membranes, and the MSCPM device has potential merit. ${ }^{12}$

To address limitations of the MSCPM-II, a smooth luminal surface and antimigration flaps were added to the new MSCPM-

\footnotetext{
Correspondence to: Dong Ki Lee ${ }^{a}$ and Sugeun Yang ${ }^{b}$

${ }^{a}$ Department of Internal Medicine, Gangnam Severance Hospital, Yonsei University College of Medicine, 211 Eonju-ro, Gangnam-gu, Seoul 06273, Korea

Tel: +82-2-2019-3214, Fax: +82-2-3463-3882, E-mail: dklee@yuhs.ac

${ }^{\text {b} W o r l d ~ C l a s s ~ S m a r t ~ L a b, ~ D e p a r t m e n t ~ o f ~ N e w ~ D r u g ~ D e v e l o p m e n t, ~ I n h a ~ U n i v e r s i t y ~ C o l l e g e ~ o f ~ M e d i c i n e, ~} 100$ Inha-ro, Michuhol-gu, Incheon 22212, Korea

Tel: +82-32-890-2832, Fax: +82-32-890-1199, E-mail: sugeun.yang@inha.ac.kr
}

Received on October 6, 2018. Revised on November 16, 2018. Accepted on November 18, 2018. Published online April 17, 2019

pISSN 1976-2283 eISSN 2005-1212 https://doi.org/10.5009/gnl18454

(a) This is an Open Access article distributed under the terms of the Creative Commons Attribution Non-Commercial License (http://creativecommons.org/licenses/by-nc/4.0) which permits unrestricted non-commercial use, distribution, and reproduction in any medium, provided the original work is properly cited. 
III stent. Also, sodium caprate (SC) was included as a penetration enhancer to promote local antitumor effect and thus discourage occlusive tumor ingrowth. SC has been added to augment cancerous tissue penetration by PTX eluted from the MSCPM-III stent. ${ }^{13} \mathrm{SC}$ is an agent known to heighten absorption of various drugs, such as ampicillin, insulin, low-molecular-weight heparin, and 5-fluorouracil. ${ }^{14}$ This study was conducted to evaluate the safety of the newly developed third-generation MSCPM-III stent in a porcine model, determining the feasibility of human application.

\section{MATERIALS AND METHODS}

\section{Metal stent incorporating PTX- and SC-impregnated membranes}

Structural modifications were made to prevent early stent occlusion or migration. The MSCPM-II design is such that a membrane (Fig. 1A, b) is positioned between two layers of metal mesh (Fig. 1A, a). Thus, the luminal surface could become kinked and easily clogged by sludge or food (Fig. 1C). In the MPCPM-III stent, a layer of metal mesh is placed between two membranes (Fig. 1B) for a smooth lumen (Fig. 1D), and flaps were placed on both sides to prevent stent migration (Fig. 1E). The stent used for animal experiments was $6 \mathrm{~mm}$ in diameter and $2 \mathrm{~cm}$ in length when fully expanded. The delivery catheter used for animal experiments was 8.5 Fr, and the diameter of the stent was $2.8 \mathrm{~mm}$ before expansion and $6 \mathrm{~mm}$ at maximum expansion. After expanding the stent, the maximum expanding diameter was $6 \mathrm{~cm}$ in diameter and $2 \mathrm{~cm}$ in length, so the crosssectional area was about $376 \mathrm{~mm}^{2}$.

The membrane in this study has the same double-layer structure as that described previously. ${ }^{7}$ In the MPCPM-III stent, a layer of metal mesh was placed between two membranes (inner membrane, polytetrafluoroethylene [PTFE]; outer membrane,
PU). The inner luminal aspect is composed of PTFE, which is resistant to bile acid, and the outer membrane is made of PU (Pellethane 2363-80A; Lubrizol Corp, Wickliffe, OH, USA). In addition to PTX $0.27 \mathrm{mg} / \mathrm{cm}^{2}$, SC (Sigma-Aldrich, St Louis, MO, USA) has been added to augment cancerous tissue penetration by PTX (Samyang Biopharmaceuticals, Seoul, Korea) eluted from the MSCPM-III stent. ${ }^{13}$ The MSCPM-III stent coating consists of $400 \mathrm{mg}$ (10\%) PTX-SC-PU polymer and $10 \mathrm{~mL}$ tetrahydrofuran (THF) solution (Junsei Chemical, Tokyo, Japan) (PTX 40 mg+SC 40 mg+PU 320 mg+THF 10 mL). Control stents were coated with SC-PU polymer and THF (10 mL) only (SC 40 $\mathrm{mg}+\mathrm{PU} 360 \mathrm{mg}+\mathrm{THF} 10 \mathrm{~mL}$ ). The coating process involves a rolling technique.

\section{Drug release profile}

This study used a stent that has been reported previously. ${ }^{13}$ In a previous study, ${ }^{13}$ stents coated with different amounts of SC (0, $0.15,0.32$, and $\left.0.51 \mathrm{mg} / \mathrm{cm}^{2}\right)$ and a fixed amount $\left(0.27 \mathrm{mg} / \mathrm{cm}^{2}\right)$ of PTX were placed in $0.1 \%$ Tween 20-containing phosphatebuffered saline solution and were incubated at $37^{\circ} \mathrm{C}$ in a shaking water bath at $50 \mathrm{rpm}$ for 2 months to confirm the PTX-releasing behavior. The stent with PUSC10-PTX (SC $0.32 \mathrm{mg} / \mathrm{cm}^{2}$, PTX $0.27 \mathrm{mg} / \mathrm{cm}^{2}$ ) exhibited optimal efficacy for drug penetration into the tumor, and thus it was applied in this animal study. The amount of PTX released was quantified using an HPLC-UV system. The HPLC-UV analysis was performed at room temperature using a Thermo Scientific Hypersil C18 column (150×4.6 mm; Thermo Electron Co., Altrincham, UK). HPLC-grade methanol was the mobile phase delivered at a flow rate of $1 \mathrm{~mL} / \mathrm{min}$. PTX was detected at $227 \mathrm{~nm}$.

The drug release profile was as follows. The stent was initially loaded with $0.27 \mathrm{mg} / \mathrm{cm}^{2}$ PTX, and the amount released in vitro for 2 months was measured. The total amount of PTX released over 2 months was $0.21 \pm 0.01 \mathrm{mg} / \mathrm{cm}^{2}$ (mean \pm standard
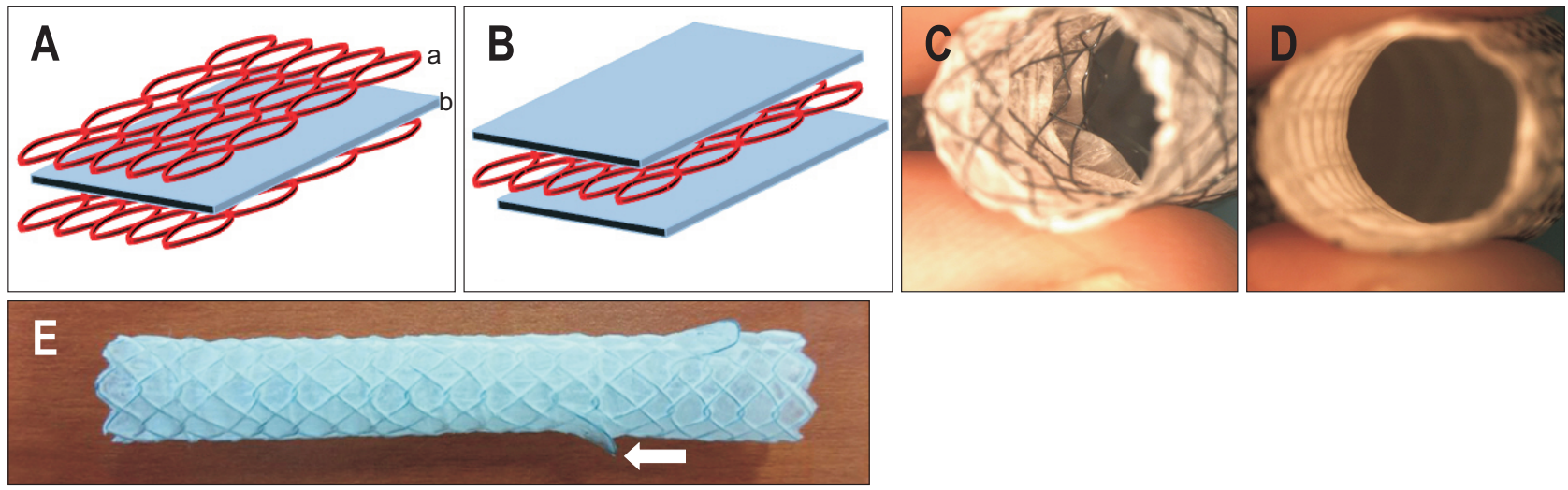

Fig. 1. Modification of metallic stent incorporating paclitaxel-impregnated membrane (MSCPM-III). (A) The previous MSCPM-II stent has two layers of metal mesh (a) with a single interposed membrane (b) leaving luminal mesh exposed. (B) In the MSCPM-III stent, metal mesh is flanked by two membranes, with no luminal mesh exposed. (C) Luminal roughness of the MSCPM-II stent encourages occlusion by sludge or food. (D) Smooth luminal surface of the MSCPM-III is less inclined to promote occlusion. (E) One of two flaps (white arrow) attached to the proximal aspect of the stent as an antimigration measure. 
deviation) PTX. The amount of PTX retained in the stent after 2 months was $0.06 \pm 0.01 \mathrm{mg} / \mathrm{cm}^{2}$. Approximately $20 \%$ of the initial burst release of PU with PTX (PU-PTX) and PU with SC and PTX (PUSC-PTX) was detected at 2 days. After 2 days, approximately $2.5 \mu \mathrm{g} / \mathrm{cm}^{2}$ PTX was released daily from the PU-PTX and PUSC-PTX membranes. Secretion by the PTX-containing membrane was similar regardless of whether SC was added.

\section{Experimental animals}

Ten mini pigs (Sus scrofa domesticus, miniature pig, micropig) 12 months old and of average weight (40 kg [ $\pm 20 \%])$ were used as test $(n=5)$ or control animals $(n=5)$. Fasting was imposed 24 hours before endoscopically advancing a stent into the bile duct of each animal. Anesthesia was induced by intravenous injection of ketamine hydrochloride (Yuhan Co, Seoul, Korea) and atropine sulfate (Daewon Pharmaceutical Co, Seoul, Korea), using ketamine hydrochloride for maintenance during the procedure. Each animal was placed in left lateral decubitus position, orally inserting the stent to the level of ampulla via $3.8-\mathrm{mm}$ retrograde pancreatic endoscope (ED-3630T video duodenoscope; Pentax Medical, Tokyo, Japan). Endoscopic clipping of the major duodenal papilla was performed using a conventional endoscope (GIF-Q260J; Olympus, Tokyo, Japan) with a fitted transparent cap (D-201; Olympus) as previously reported. ${ }^{15}$ The endoscope was positioned close to the major duodenal papilla such that the transparent cap was in contact with the papilla. The major papilla was partially ligated with one hemoclip (HX610-090L; Olympus) to prevent potential stent migration, and radiographs were taken to document stent placement. As the stent was inserted at the mid-CBD level, the distal migration of the stent from the upper side of the ampulla was confirmed by follow-up X-rays. Radiographs were obtained both immediately after stenting and after the stent was fully expanded. The estimated diameters of the stents were $2.65 \pm 0.68 \mathrm{~mm}$ immediately after stenting and $5.74 \pm 0.36 \mathrm{~mm}$ after the stent was sufficiently expanded. Afterwards, antibiotics are administered for 3 days, and the animals were fed a standard diet for 4 weeks. The pigs were monitored daily for clinical symptoms and signs of death/ dying. Body weight was measured twice weekly at acquisition, assuming a once-weekly schedule during the purification period, upon group assignment, before stent insertion, during the test period, and at the end of testing.

\section{Extraction of specimens}

After stenting and 4 weeks of monitoring, the animals were euthanized using a bolus of potassium chloride solution so that stents implanted endoscopically (including sites of insertion) could be studied. The pigs were anesthetized with $25 \mathrm{mg} / \mathrm{mL}$ tiletamine and $25 \mathrm{mg} / \mathrm{mL}$ zolazepam in a combination medication (Zoletil; Virbac, Carros, France) administered intramuscularly. Anesthesia was maintained by inhalation of isoflurane. The abdomen was opened to confirm the position of the duodenum. Stent-implanted bile ducts were sutured to the proximal portion of liver, marking the proximal aspect and leaving the stents intact. After fixation in 10\% buffered neutral formalin and routine processing, histologic sections were prepared for examination.

All experimental procedures were performed in accordance
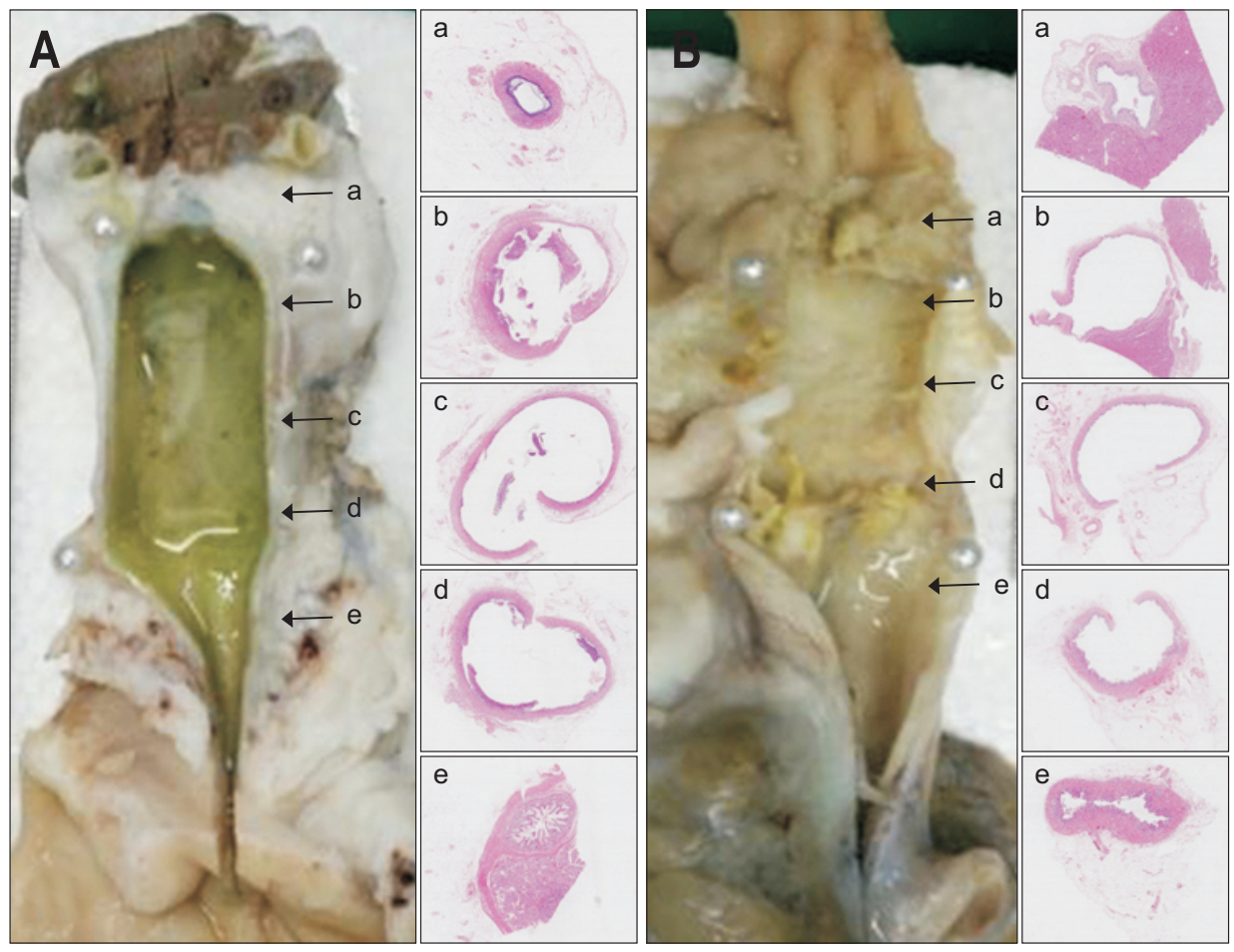

Fig. 2. Histology of extracted common bile ducts showing similar inflammatory and fibrotic reactions after stenting with $0 \%$ paclitaxel (A) or 10\% paclitaxel (B). Gross appearance (left panel) and corresponding microscopic views (right panel: H\&E, $\times 12.5$ ), arrows indicate areas sampled for slide preparation. Proximal (a) and distal (e) portions of non-stented areas retain villous mucosal pattern in both groups, whereas proximal (b), central (c), and distal (d) portions of stented areas display luminal dilatation, mucosal atrophy, and attenuated walls. 
with rules of the Institutional Animal Care Committee and were approved by the Committee on Animal Research at Inha University (permit number: MK-IACUC 140620-001) and the Animal Protection Committee of the South Korean government. All surgery was performed under sodium pentobarbital anesthesia, and all efforts were made to minimize suffering.

\section{Histologic analysis}

Formalin-fixed common bile ducts were cross-sectioned at five levels and photographs were taken to document gross appearances of proximal and distal non-stented segments (a and e, left panel in Fig. 2A and B) and proximal, central, and distal stented segments (b, c, and d, left panel in Fig. 2A and B). A single pathologist evaluated the thickness of the epithelial layers, the degree of submucosal inflammatory cell infiltration and fibrotic accumulation in extracted tissues by hematoxylin and eosin staining (H\&E). The thickness of each epithelial layer was defined as the distance between tissue protruding into the lumen and the lower portion of the submucosa. The basic cell types (i.e., monocytes), macrophages and foam cells were evaluated by $\mathrm{H} \& \mathrm{E}$ staining.

\section{Serum analysis}

Blood samples were collected at 14 and 28 days after stent implantation for biochemical testing, immediately separating the serum by centrifugation $\left(3,000 \mathrm{rpm}, 10\right.$ minutes, $\left.4^{\circ} \mathrm{C}\right)$. Lev- els of albumin, alkaline phosphatase, aspartate transaminase, alanine transaminase, total protein, total bilirubin, and direct bilirubin were measured.

Liquid chromatography was performed in tandem with mass spectrometry (LC-MS/MS) to determine serum PTX concentration. The calibration curve was prepared by adding docetaxel as internal standard to a PTX stock solution, and measuring various concentrations $(5,20,50,100,200,500$ and 1,000 ng/ $\mathrm{mL}$ ) in the same manner as blood analysis. Pretreatment of the blood was performed by solvent extraction method as follows: blood plasma $(10 \mu \mathrm{L})$ and mobile phase $(10 \mu \mathrm{L})$ containing the internal standard were vortexed; ethyl acetate $(250 \mu \mathrm{L})$ was added to the blood sample and shaken, followed by centrifugation (14,000 rpm); and after centrifugation, the supernatant was decanted and dried, and $20 \mu \mathrm{L}$ of mobile phase was added. The calibration curve thus obtained was linear in the test range and was $y=0.0126 \times-0.0286\left(R^{2}=0.992\right)$.

\section{Statistical analyses}

Quantified serum analytes were compared using repeated ANOVA, and the results are expressed as means \pm standard deviation. All computations relied on SPSS version 12.0 (SPSS Inc, Chicago, IL, USA), setting significance at $\mathrm{p}<0.05$.
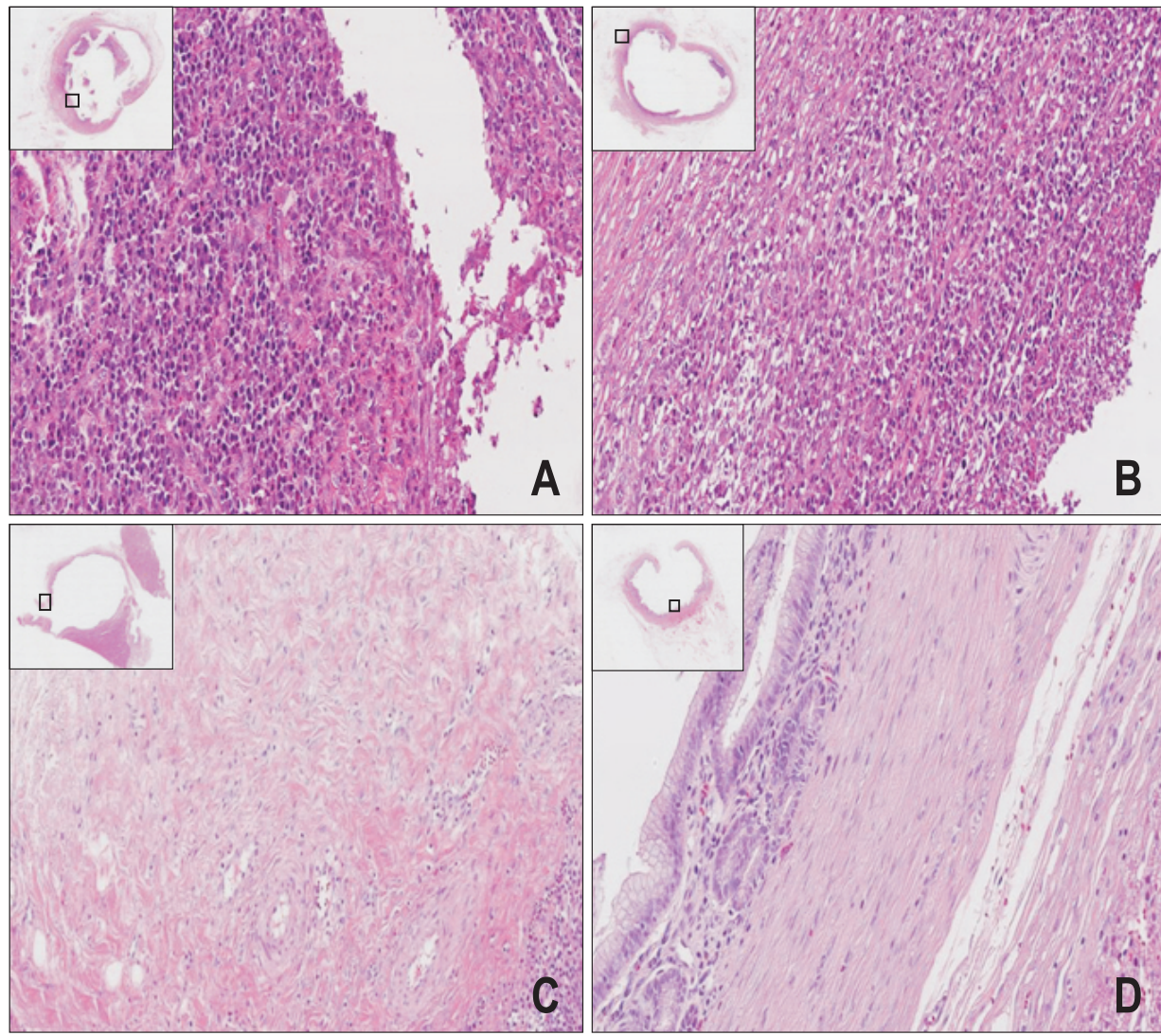

Fig. 3. Common bile ducts (H\&E, $\times 12.5$ ) showing histologic similarities (luminal dilatation, mucosal atrophy, and attenuated walls) of both the control (A, B) and test groups (C, D), including damaged mucosal layers and denuded areas in contact with stents. (A, B) Dense cellularity of bile duct, with sharp nuclear staining across the top of areas in contact with stents (control specimen); and (C, D) loss of nuclear membranes and patchy fibrosis of the muscular layer across the bottom of areas in contact with stents (test specimen); no necrosis or perforation of bile duct serosa. 


\section{RESULTS}

\section{Outcome after stent insertion}

One occurrence each of stent migration and obstructive jaundice were recorded in the test group, and there were two stent migrations in the control group. The isolated instance of obstructive jaundice developed on day 10 after stent placement as a consequence of biliary ligation (during endoscopic stenting) to prevent stent migration. However, food impaction or sludge-induced stent occlusion was not observed. The episode of stent migration occurred 2 weeks after insertion. However, no perforation or necrosis of bile duct occurred due to stenting. The final analysis was based on three test animals and three control animals.

\section{Between-group histologic comparison}

Stented areas (b, c, and d, left panel in Fig. 2A and B) had visibly larger luminal diameters and thinner walls than nonstented areas (a and e, left panel in Fig. 2A and B), and mucosal surfaces in stented areas were flat and atrophic. Nevertheless, no perforation or necrosis was observed. These findings were comparable in both groups and were expected due to stent compression of bile duct walls.

Microscopic examination of control stents showed that the mucosal lining of biliary duct was denuded (Fig. 3A and B) at areas in contact with stents, presumably a mechanical effect of stenting. This was true of both groups. In non-stented areas, mild-to-moderate inflammation, limited or no fibrosis, and varying degrees of mucosal sloughing or atrophy were observed. However, in PTX-eluting stents, the nuclear membranes of cells had disappeared, and patchy fibrosis was observed involving muscular layer of bile duct wall (Fig. 3C and D). This was presumptive cytotoxic effect of eluted PTX, intended to delay stent infiltration by bile duct cancer.

\section{Serum analysis}

Serum biochemical tests at the 2- and 4-week marks did not differ significantly in either group, nor were between-group differences significant at these points in time (Fig. 4). In LC-MS/ MS analysis, the amount of liberated PTX in serum was reported as limit of detection.

\section{DISCUSSION}

Although the first-generation MSCPM-I stent was found stable and effective in relieving malignant biliary obstruction during animal ${ }^{5}$ and preliminary human ${ }^{8}$ investigations, a comparator study in conjunction with non-drug-eluting covered metal stents did not confirm its efficacy. ${ }^{10}$ It seemed that tumor ingrowth was fostered by micro-imperfections and holes in the
A

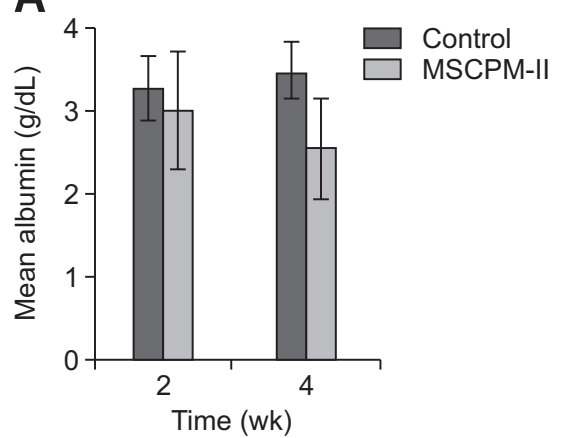

D

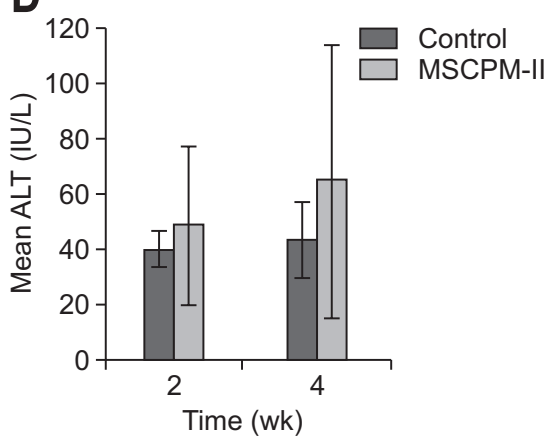

B

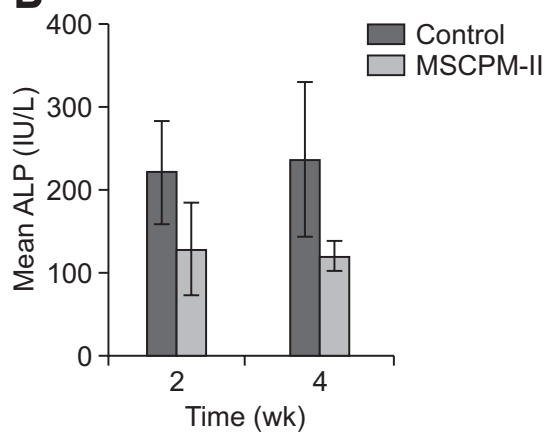

$E$

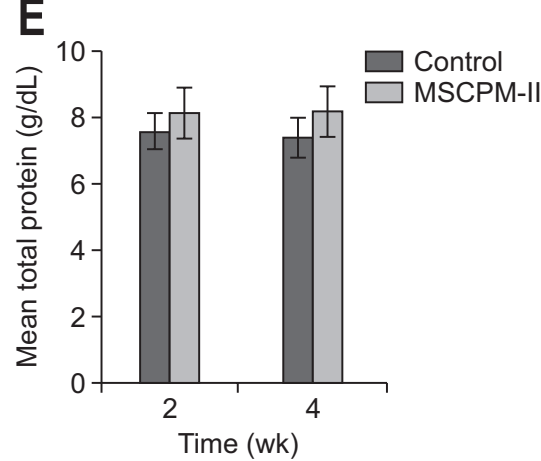

C
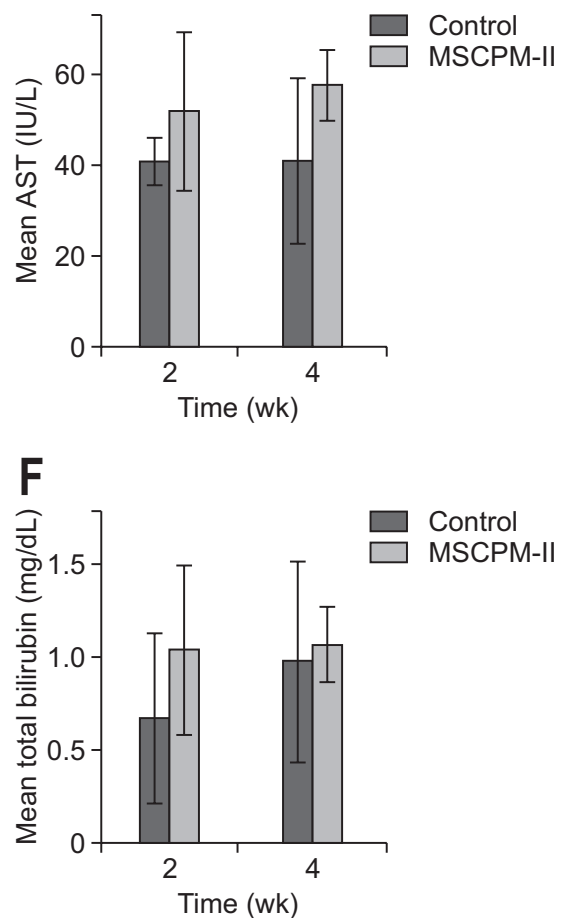

Fig. 4. Biochemical testing of serum: albumin (A), ALP (B), AST (C), ALT (D), total protein (E), and total bilirubin (F) measured at 2 and 4 weeks after stent implantation. The results were presented as the mean \pm SD. No significant differences in group outcomes were found between each measurement at 2 and 4 weeks.

ALP, alkaline phosphatase; AST, aspartate transaminase; ALT, alanine transaminase. 
PU membrane, and that PTX release was insufficient to ensure antitumor effect. To overcome these limitations, strategic utilization of PTFE in the second-generation MSCPM-II device served to prevent biodegradation by bile acids, and another layer of PU was added. ${ }^{7}$ Pluronic F-127 was also applied to prevent tumor ingrowth and progression through continuous, long-term release of high PTX concentrations. Again, the MSCPM-II was found safe in a prospective comparative human study, tested alongside conventional covered metal stents, but its efficacy could not be confirmed due to early stent occlusion (by food or sludge) or stent migration. ${ }^{11}$ The rough luminal surface of the MSCPM-II stent, rather than tumor ingrowth, was responsible for this problematic early occlusion.

As a third-generation device, the MSCPM-III stent was structured differently, sandwiching a core of metal mesh between two membranes to impart a smooth luminal surface. Antimigration flaps were also mounted on both sides to prevent migration of the covered SEMS, and SC was added to enhance penetration of PTX and boost antitumor effect. MSCMP-III is the same stent that has been reported previously. ${ }^{13}$ In the PTX-SC-PU membrane, PTX was found to release for 60 days through in vitro drug release testing, without an initial burst. ${ }^{13}$ Long-term and continuous PTX release was achieved without Pluronic F-127 by changing the drug-eluting membrane production method from a dipping method to a rolling technique. Ultimately, the penetration depth of PTX increased significantly in vivo and in vitro by adding SC. ${ }^{13}$

Biliary ductal attenuation, mild-to-moderate inflammation, limited or no fibrosis, and varying degrees of mucosal sloughing or atrophy in stented areas including anti-migration flap lesion were findings in MSCPM-III at this time, corresponding with those of previous animal studies. ${ }^{5,7}$ On the other hand, mucinous metaplasia and bile duct thickening observed in animal testing of the MSCPM-I stent ${ }^{5}$ and intestinal metaplasia developing during animal study of the MSCPM-II stent ${ }^{7}$ were not manifested in MSCPM-III-exposed tissues. Furthermore, the histological changes seen after MSCPM-I and MSCPM-II placement were confined to the mucosal layer, whereas fibrosis in the deep muscle layer of the bile duct was apparent following MSCPM-III placement, indicating that PTX penetrated further using the MSCPM-III (enhanced by SC), even though all devices contained identical PTX concentrations. However, because we performed this study in normal bile ducts and did not evaluate the concentration of PTX in extracted bile ducts, we could not confirm the effectiveness of SC as a penetration enhancer. Nevertheless, the serosa was not necrotic, and the fibrosis observed in deep muscle may be beneficial by acting as a physical barrier to tumor ingrowth, enhancing the localized inhibition of tumor invasion by PTX.

The antimigration flaps attached to MSCPM-III stents were concerning. Despite this addition, the migration rate averaged 30\%: 20\% (1/5 cases) in the test group and 40\% (2/5 cases) in the control group. Although the MSCPM-III stent had a significantly lower migration rate than the control stent, we cannot safely predict the migration rate in clinical practice because we used a pig model with normal bile ducts. In clinical settings, the migration rate would be expected to be lower because most patients will exhibit biliary strictures. By comparison, there were no instances of stent migration in animal testing of the MSCPM-II stents. ${ }^{7}$ One reason for such disparity is that the MSCPM-II stents were surgically inserted into bile ducts, leaving ampulla intact but likely immobilizing the devices through incisional scarring and focal stenosis. Although migration was not encountered during animal testing, it did occur in clinical trials. ${ }^{11}$ On the other hand, when inserting the MSCPM-III stent after endoscopic cannulation of ampulla and sphincterotomy, the stent tends to easily migrate through ampulla.

Another explanation is that the radial force of the MSCPMIII stent is weaker than that of the double-layered MSCPM-II. Low radial force of SEMS might be risk factor of migration. ${ }^{16}$ The CBD diameters of mini pigs can be regarded as 3 to $4 \mathrm{~mm}$ in vivo. ${ }^{15}$ As in previous studies, ${ }^{5-7}$ the stents used in this study were $6 \mathrm{~mm}$ in diameter so that the diameter of stents might be suitable for bile ducts of experiment animals in this study. This appeared to be the result of a weakening of the stent radial force after modifying the stent structure. It is considered that the radial force of the single-layer structured MSCPM-III was reduced compared with that of the double-layer structured MSCMP-II. Such weakness could be a drawback in fixing the stent to bile duct. Finally, the smooth outer surface of the MSCPM-III device naturally may result in more migrations. Therefore, both the radial force and the antimigration flaps may need to be improved before further applications in human studies. Once modified, the migration rate in malignant biliary stenosis (unlike normal porcine duct) is expected to be low. Although the stent migration rate of the MSCPM-III did not reduce, the stent was not occluded by impacted food or sludge. In addition, severe complications did not occur in the bile duct. Thus, the MSCPM-III is safe and can be confidently placed in humans.

If we anticipated stent migration in an animal after deployment, the ampulla was partially ligated using hemoclips. One of the pigs subjected to ligation later developed obstructive jaundice and expired as an unfortunate and unavoidable complication. An alternative porcine biliary stricture model, using heat probes and radiofrequency ablation, ${ }^{17}$ was inappropriate for our purposes, given the detrimental effects on biliary histology.

PTX concentration was assayed and various biochemical tests were performed to determine the systemic impact of its release by MSCPM-III stents. However, serum levels of PTX were too low for detection, confirming that only surrounding tissues are penetrated, with no systemic effects. In a previous human study involving $10 \% \mathrm{PTX}^{8}$ low levels of PTX $\left(1.5 \times 10^{-2}\right.$ to $2.0 \times 10^{-1}$ $\mathrm{mg} / \mathrm{mL}$ ) was detected in the blood samples of patients for over 50 days, but levels approximated the therapeutic range of PTX 
$\left(0.9 \times 10^{-4}\right.$ to $\left.1.1 \times 10^{-1} \mathrm{mg} / \mathrm{mL}\right)$, and there were no systemic effects of PTX. In the present study, biochemical analysis was performed to monitor PTX hepatotoxicity, but no abnormalities were identified in either the test group or in controls.

There were some limitations in this study. In this study, the PTX level in serum was measured, but the level released into the bile duct or remaining in the bile duct, urine, and feces could not be measured. As no PTX was released into the blood, it was not expected in urine or feces, but a limitation of the study is that we did not obtain all pharmacokinetic measurements. In the previous study, ${ }^{13}$ the release rate of PTX was high in the first few days; therefore, serum testing should have been performed during this period. However, in our animal experiment, the serum test proceeded at 2 weeks and there was a discrepancy between the in vitro and in vivo experiments. Although no fatal systemic complications occurred during the 4-week observation period, a limitation was that a systemic response was not confirmed at the time of the greatest release of PTX. Although the efficacy of SC in cancer has been documented in a previous study, ${ }^{13}$ we did not compare MSCPM-III (stent with PTX and SC) and MSCPM-III without SC. Therefore, we could not compare the effectiveness of SC as a penetration enhancer. Furthermore, because the present study was conducted in normal bile ducts, we could not confirm penetration of PTX into cancer tissue by SC. The uptake of PTX by penetration of SC may be different between normal and malignant bile ducts due to differences in physical properties (hydrophobicity, lipid content, water content, etc.) of the tissues; therefore, further studies are required. Based on these studies, it is expected that the anti-tumoral effect of PTX with penetration of SC on malignant bile ducts will be more clearly demonstrated.

In conclusion, drug-eluting stents designed to relieve malignant biliary obstruction have been modified and improved through the aid of animal studies and human clinical trials. The MSCPM-III stent with SC was developed to overcome the limitations of its drug-eluting predecessors. This study confirms the histologic and systemic stability of the MSCPM-III stent in the porcine bile duct. Clinical studies are now needed to validate its safety and efficacy in patients.

\section{CONFLICTS OF INTEREST}

No potential conflict of interest relevant to this article was reported.

\section{ACKNOWLEDGEMENTS}

This work was supported by the following programs: (1) the Technology Innovation Program (10044021, Development of nonvascular drug eluting stent for treatment of gastrointestinal disease), funded by the Ministry of Trade, Industry \& Energy (MI, Korea); (2) Grants from the Korean Heath Industry Development
Institute (KHIDI) and the National Center of Efficacy Evaluation for the Development of Health Products Targeting Digestive Disorders (NCEED); and (3) the Basic Science Research Program through the National Research Foundation of Korea (NRF) (2017R1A2A2A07001272) and a World Class Smart Lab (WCSL) research grant from Inha University.

\section{AUTHOR CONTRIBUTIONS}

Drafting of the manuscript: S.I.J., D.K.L. Critical revision of the manuscript for important intellectual content: S.I.J., S.J., D.H.L., K.N., S.Y., D.K.L. Statistical analysis: S.I.J., K.N., S.Y., D.K.L. Data acquisition, data analysis and interpretation: S.J., K.N., S.Y. Administrative, technical, or material support: D.H.L., S.Y. Study concept and design: S.Y., D.K.L. Obtained funding, study supervision: D.K.L.

\section{ORCID}

Sung Ill Jang

https://orcid.org/0000-0003-4937-6167

Seok Jeong https://orcid.org/0000-0001-6178-8338

Don Haeng Lee https://orcid.org/0000-0003-0397-302X

Kun Na

Sugeun Yang

https://orcid.org/0000-0002-9629-0289

Dong Ki Lee

https://orcid.org/0000-0001-5278-8723

https://orcid.org/0000-0002-0048-9112

\section{REFERENCES}

1. Andersen JR, Sørensen SM, Kruse A, Rokkjaer M, Matzen P. Randomised trial of endoscopic endoprosthesis versus operative bypass in malignant obstructive jaundice. Gut 1989;30:1132-1135.

2. Wagner HJ, Knyrim K, Vakil N, Klose KJ. Plastic endoprostheses versus metal stents in the palliative treatment of malignant hilar biliary obstruction. A prospective and randomized trial. Endoscopy 1993;25:213-218.

3. Yoon WJ, Lee JK, Lee KH, et al. A comparison of covered and uncovered Wallstents for the management of distal malignant biliary obstruction. Gastrointest Endosc 2006;63:996-1000.

4. Telford JJ, Carr-Locke DL, Baron TH, et al. A randomized trial comparing uncovered and partially covered self-expandable metal stents in the palliation of distal malignant biliary obstruction. Gastrointest Endosc 2010;72:907-914.

5. Lee DK, Kim HS, Kim KS, et al. The effect on porcine bile duct of a metallic stent covered with a paclitaxel-incorporated membrane. Gastrointest Endosc 2005;61:296-301.

6. Lee SS, Shin JH, Han JM, et al. Histologic influence of paclitaxeleluting covered metallic stents in a canine biliary model. Gastrointest Endosc 2009;69:1140-1147.

7. Jang SI, Kim JH, Kim M, et al. Porcine feasibility and safety study of a new paclitaxel-eluting biliary stent with a Pluronic-containing membrane. Endoscopy 2012;44:825-831.

8. Suk KT, Kim JW, Kim HS, et al. Human application of a metallic 
stent covered with a paclitaxel-incorporated membrane for malignant biliary obstruction: multicenter pilot study. Gastrointest Endosc 2007;66:798-803.

9. Song TJ, Lee SS, Yun SC, et al. Paclitaxel-eluting covered metal stents versus covered metal stents for distal malignant biliary obstruction: a prospective comparative pilot study. Gastrointest Endosc 2011;73:727-733.

10. Jang SI, Kim JH, You JW, et al. Efficacy of a metallic stent covered with a paclitaxel-incorporated membrane versus a covered metal stent for malignant biliary obstruction: a prospective comparative study. Dig Dis Sci 2013;58:865-871.

11. Jang SI, Lee SJ, Jeong S, et al. Efficacy of a multiplex paclitaxel emission stent using a Pluronic ${ }^{\circledR}$ mixture membrane versus a covered metal stent in malignant biliary obstruction: a prospective randomized comparative study. Gut Liver 2017;11:567-573.

12. Bang S, Jang SI, Lee SY, et al. Molecular mechanism of local drug delivery with Paclitaxel-eluting membranes in biliary and pancreatic cancer: new application for an old drug. Gastroenterol Res Pract 2015;2015:568981.
13. Jeong D, Lee DH, Lee DK, Na K. Nonvascular drug-eluting stent coated with sodium caprate-incorporated polyurethane for the efficient penetration of paclitaxel into tumor tissue. J Biomater Appl 2015;29:1133-1144.

14. Fujioka T, Kondou T, Fukuhara A, et al. Efficacy of a glycyrrhizin suppository for the treatment of chronic hepatitis C: a pilot study. Hepatol Res 2003;26:10-14.

15. Park JS, Kwon CI, Jeong S, Kim K, Moon JH, Lee DH. Development of a swine bile duct dilation model using endoclips or a detachable snare under cap-assisted endoscopy. Gastrointest Endosc 2014;80:325-329.

16. Nakai Y, Isayama H, Kogure H, et al. Risk factors for covered metallic stent migration in patients with distal malignant biliary obstruction due to pancreatic cancer. J Gastroenterol Hepatol 2014;29:1744-1749.

17. Shin JU, Lee KH, Kim SA, et al. Intraductal thermal injury using a heat probe and radiofrequency ablation electrode in a swine model of biliary stenosis. Clin Res Hepatol Gastroenterol 2013;37:159165. 